\title{
Aging alters the molecular dynamics of synapses in a sexually dimorphic pattern in zebrafish (Danio rerio)
}

\author{
Elif Tugce Karoglu a,b,c , Dilara Ozge Halim ${ }^{\mathrm{d}}$, Bahriye Erkaya ${ }^{\mathrm{d}}$, Ferda Altaytas ${ }^{\mathrm{d}}$, \\ Ayca Arslan-Ergul ${ }^{\text {a,b,c }}$, Ozlen Konu ${ }^{\text {a,b,c,d }}$, Michelle M. Adams ${ }^{\text {a,b,c,e,* }}$ \\ anterdisciplinary Graduate Program in Neuroscience, Aysel Sabuncu Brain Research Center, Bilkent University, Ankara, Turkey \\ ${ }^{\mathrm{b}}$ UNAM-Institute of Materials Science and Nanotechnology, Bilkent University, Ankara, Turkey \\ ${ }^{\mathrm{c}}$ Department of Molecular Biology and Genetics Zebrafish Facility, Bilkent University, Ankara, Turkey \\ ${ }^{\mathrm{d}}$ Department of Molecular Biology and Genetics, Bilkent University, Ankara, Turkey \\ ${ }^{\mathrm{e}}$ Department of Psychology, Bilkent University, Ankara, Turkey
}

\section{A R T I C L E I N F O}

\section{Article history:}

Received 8 August 2016

Received in revised form 9 February 2017

Accepted 10 February 2017

Available online 20 February 2017

\section{Keywords:}

Excitatory synapses

Inhibitory synapses

Synaptic proteins

Aging

Gender

Zebrafish

\begin{abstract}
A B S T R A C T
The zebrafish has become a popular model for studying normal brain aging due to its large fecundity, conserved genome, and available genetic tools; but little data exists about neurobiological age-related alterations. The current study tested the hypothesis of an association between brain aging and synaptic protein loss across males and females. Western blot analysis of synaptophysin (SYP), a presynaptic vesicle protein, and postsynaptic density-95 (PSD-95) and gephyrin (GEP), excitatory and inhibitory postsynaptic receptor-clustering proteins, respectively, was performed in young, middle-aged, and old male and female zebrafish (Danio rerio) brains. Univariate and multivariate analyses demonstrated that PSD-95 significantly increased in aged females and SYP significantly decreased in males, but GEP was stable. Thus, these key synaptic proteins vary across age in a sexually dimorphic manner, which has been observed in other species, and these consequences may represent selective vulnerabilities for aged males and females. These data expand our knowledge of normal aging in zebrafish, as well as further establish this model as an appropriate one for examining human brain aging.
\end{abstract}

(C) 2017 Elsevier Inc. All rights reserved.

\section{Introduction}

Aging is accompanied by a range of variations in cognitive processing, which are likely due to subtle biological alterations in the brain structures that underlie these behaviors. Initial studies indicated that neuronal loss contributed to these changes (Brody, 1955; Colon, 1972; Dayan, 1970), but subsequent work has demonstrated that significant cell loss is not present in the aged brain (Rapp and Gallagher, 1996; Rasmussen et al., 1996; West et al., 1991). Interestingly, it appears that global synapse loss does not seem to be occurring in the aging brain (Calhoun et al., 1998; Newton et al., 2008; Poe et al., 2001; Shi et al., 2007). Therefore, subtle changes in synaptic structure or composition, which alter synaptic plasticity and functional responses, are most likely contributing to synaptic dysfunction in the aging brain (Adams et al., 2008; Ganeshina et al., 2004; VanGuilder et al., 2010, 2011). Thus, understanding what these alterations are and how they can

\footnotetext{
* Corresponding author at: Interdisciplinary Graduate Program in Neuroscience, Aysel Sabuncu Brain Research Center, Bilkent University, 06800 Bilkent, Ankara, Turkey. Tel.: 90312290 1090; fax: 903122902561.

E-mail address: michelle@bilkent.edu.tr (M.M. Adams).
}

be corrected is an important goal for altering the course of cognitive decline.

In addition to the effects of age on synaptic structure and composition, it is well established that there are brain areas underlying age-related cognitive decline that are responsive to sex hormones. In the hippocampus, changes in circulating estrogen and progesterone levels alter synaptic structure (Adams et al., 2001; Woolley and McEwen, 1993) and composition in female rats (Adams et al., 2001), and these brain areas respond differentially to hormones in young and aged animals (Adams et al., 2001). Moreover, there are sex differences in hippocampal synaptic responses to circulating hormones in rats (Brandt et al., 2013). Despite this knowledge, most of the studies examining age-related synaptic changes have been done in male animals. However, since there are sex differences in synaptic structure and function and brain areas respond differentially to sex hormones, it is important to determine whether male and female brains vary with age in a similar manner.

The zebrafish (Danio rerio) has recently come into favor as a useful and appropriate model organism for examining age-related changes in behavior and biology due to its large fecundity, conserved genome, and genetic tools that are readily available. Zebrafish exhibit advanced behavioral properties like spatial 
memory and social behaviors similar to those described in other mammals (Kishi et al., 2009; Lieschke and Currie, 2007). Moreover, the integrated nervous system of the zebrafish brain has been proposed to contain homologous structures to those of other mammals (Friedrich et al., 2010; Wulliman et al., 1996). For example, the ventrolateral portion of the dorsal telencephalic area, which is the medial pallium, has been proposed to correspond to the mammalian hippocampus (Friedrich et al., 2010; Ganz et al., 2014). Functional studies have demonstrated that lesions in this region lead to impairments in spatial learning (Rodriguez et al., 2002). Moreover, the medial zone of the dorsal telencephalic area, which is the ventral/latero pallium, has been proposed to be homologous to the mammalian amygdala (Friedrich et al., 2010; Ganz et al., 2014). Ablation studies of this structure have revealed impairments in fear conditioning (Friedrich et al., 2010). Another distinct region in the zebrafish brain is the optic tectum (TeO) which has been proposed to be similar to motion processing areas in mammalian visual cortex due to this structure underlying firstorder and second-order motion detection (Orger et al., 2000). Thus, the behaviors zebrafish exhibit and the organization of their brains are homologous to mammals.

Zebrafish have similar patterns as mammals with regards to aging and their lifespan. On average, they live about 3 years and age gradually like mammals (Kishi et al., 2003). One study demonstrated that aged zebrafish exhibit declines in spatial memory between 1 and 3 years (Yu et al., 2006). Moreover, senescence associated ß-galactosidase, which is a biomarker of aging, increases with advancing age in zebrafish, and this also has been described in humans (Arslan-Ergul et al., 2016; Kishi et al., 2003). However, only a few studies have focused on the neurobiological consequences of aging in the zebrafish brain. They have demonstrated that gene expression levels related to processes such as neuronal differentiation as well as synaptic structure and growth are altered with age (Arslan-Ergul and Adams, 2014; Santos et al., 2008; Toyama et al., 2009), and there are decreases in neurogenesis in aged brains (Arslan-Ergul et al., 2016; Edelman et al., 2013). These data have begun to help validate the zebrafish as an appropriate model for understanding the neurobiological consequences of aging, but significant work is still required.

Differences in key synaptic protein levels can provide information about pre-/postsynaptic integrity and the excitatory/inhibitory components of neurotransmission that might be disturbed or altered during the normal aging process. Three important proteins that are markers of synaptic integrity are postsynaptic density (PSD)-95, gephyrin (GEP), and synaptophysin (SYP). PSD-95 by its PDZ domains serves as a scaffold protein that provides a docking site for signaling proteins clustering around both NMDA- and AMPA-type glutamate receptors, which are crucial for excitatory neurotransmission and synaptic plasticity (Sheng, 2001). In mutant mice, which lack PSD-95, aberrant long-term potentiation cooccurs with spatial learning and memory impairments (Migaud et al., 1998). Alternatively, in vitro studies have indicated that PSD-95 enhances long-term potentiation and long-term depression, and converts silent synapses into functional ones (Stein et al., 2003). Therefore, it can be said that PSD-95 is crucial for normal synaptic functioning and could provide information about the excitatory portion of neurotransmission due to its clustering role surrounding glutamate receptors. GEP is a core scaffolding protein, which is the postsynaptic component of inhibitory synapses, and it controls the formation and plasticity of inhibitory synapses through regulation of $\mathrm{GABA}_{\mathrm{A}}$ and glycine receptors (Tyagarajan and Fritschy, 2014). Studies conducted with GEP-deficient mice have demonstrated that this component is required for the localization of the $\mathrm{GABA}_{\mathrm{A}}$ and glycine receptors (Kneussel et al., 1999), and in vitro studies have shown that overexpression of GEP resulted in a smaller size of PSD-95 clusters and glutamatergic terminals (Wendou and De Blas, 2008). Moreover, it has major effects on experiencedependent plasticity by altering inhibitory synaptic neurotransmission (Chen et al., 2012; Dejanovic et al., 2014). Therefore, GEP levels in the aging brain would definitely provide information about the inhibitory component of neurotransmission and possibly an excitatory segment. Taken together, these data indicate that GEP could underlie synaptic dysfunction in the aging brain. SYP is an integral membrane glycoprotein found in presynaptic vesicles (Jahn et al., 1985; Wiedenmann and Franke, 1985) and it was shown to play a solitary role in endocytosis and vesicle trafficking (Kwon and Chapman, 2011). Previous studies have demonstrated that the levels of SYP change throughout the aging process and are related to cognitive impairments. For example, SYP levels are decreased in aged rats with learning deficits (Smith et al., 2000) and a significant correlation between memory performance and SYP levels has been documented (Calhoun et al., 1998). Therefore, SYP levels in the aging brain are a good indicator of presynaptic integrity and cognitive decline that occurs during normal aging.

The purpose of the current study was to test the hypothesis that there is an association between the aging zebrafish brain and synaptic protein loss across males and females as measured by levels of SYP, a presynaptic marker protein, PSD-95, a postsynaptic marker of excitatory synapses, and GEP, a postsynaptic marker of inhibitory synapses. Both genders were included because current evidence suggests that alterations in gene expression levels are differentially affected in male and female zebrafish brains (Arslan-Ergul and Adams, 2014) and neurogenesis rates are sexually dimorphic (Ampatzis et al., 2012). Our results demonstrated that the excitatory, inhibitory, and presynaptic proteins used in the present study have differential patterns of aging that depend on gender, and these are the first data to examine synaptic changes in individual zebrafish brains across different ages and genders.

\section{Methods}

\subsection{Animals}

A total of 52 wild-type zebrafish (AB strain) were used for this study. The distribution of the fish was young (6-8 months old), middle-aged (11-14.5 months old), and old (24-30 months old) male and female animals. This study was designed to determine the effects of aging from a period of young adulthood until very old based on the fact that zebrafish like rodents are defined as adultlike once they reach sexual maturity, which is approximately 3 months of age (Kimmel et al., 1995; Parichy et al., 2009; Singleman and Holtzman, 2014). There were 6 animals per group, totaling 36 animals, for protein analyses of the whole brain, and 3 animals per group, totaling 12 animals, for the microdissection experiments. In addition, a set of 4 very old female fish (34-43 month old) were added to the study for further comparisons among the groups of females. All fish were raised in standard conditions in the zebrafish facility in Bilkent University's Molecular Biology and Genetics Department, Ankara, Turkey. Fish were maintained at a constant temperature of $28.5^{\circ} \mathrm{C}$ on a 14L:10D light cycle. They were fed 2 times daily with standard fish flakes and 3 times a week with fresh artemia in a recirculating tank system. All animals were kept in groups with approximately 10 fish in a 4-L tank with minimal disruption to prevent any unnecessary stress for the animals. Based on a previous study, the stocking density used in the present experiments should not induce an increase in stress and/or cortisol levels (Pavlidis et al., 2013). Birthdates were recorded and only fish with the identical dates of birth were kept together in the same tank. These groups were maintained in the system throughout the study so as to not disrupt any social 
hierarchy. For the brain dissection, fish were anesthetized in Tricaine ( $0.3 \mathrm{~g} / \mathrm{L}$; Sigma-Aldrich, St. Louis, MO, USA) and the head was separated from the body with a scalpel. The eyes and optic nerves were detached to be able to remove the brain from the skull. Following visualization of the brain, it was removed and placed into a $0.5-\mathrm{mL}$ microtube, frozen in liquid nitrogen, and stored at $-80^{\circ} \mathrm{C}$ until protein isolation. In the first analysis, we used whole brain lysates to understand global changes in the aging male and female brains. However, to determine whether there are region-specific alterations, 3 young male and 3 young female brains, as well as 3 old male and 3 old female brains were microdissected into 3 separate regions that included: (1) telencephalon (Tel); (2) TeO; and (3) cerebellum/medulla/spinal cord (Ce). These ages were chosen because if there were any region-specific differences across lifespan, it would be expected for the largest ones to be between young and old animals. First, the whole brains were placed with the dorsal side up, and then the 3 distinct regions were visually separated using the zebrafish brain atlas as a guide (Wullimann et al., 1996). After the Tel, TeO, and Ce were separated from each whole zebrafish brain sample, the 3 microdissected pieces from each of the 3 animals in the same group were pooled together by putting them into the same tube with lysis buffer and then immediately homogenized. The result was that within those 4 age and gender groups (female-young, male-young, female-old and male-old) there was 1 pooled stock from each of the 3 brain regions, Tel, TeO, and Ce. The reason for the pooling was mainly due to the size of the Tel, which is relatively smaller than the other regions of the zebrafish brain, and would result in low total-protein concentration. The low protein recovery in a nonpooled sample would prevent obtaining a minimum of 3 replicates for each blot, which requires at least $150 \mu \mathrm{g}$ of protein from each sample. In addition, we pooled separately the $\mathrm{TeO}$ and Ce samples to accurately compare potential synaptic protein differences among these areas. During all brain dissections, internal organs including gonads were also dissected and visualized. In cases where there were eggs, the gender was designated as being female, and in cases of testes, the gender was denoted as being male. Those animals with no visible eggs or testes were not included in the study. In addition, since the antibodies used in this study have not been used or optimized previously for Western blot analysis, 1 adult mouse brain, specifically cortex, was used for validation of the molecular weight of the observed bands from the zebrafish brain samples. The animal protocol for this study was approved by the Bilkent University Local Animal Ethics Committee (HADYEK) with approval date: Feb 9, 2010 and no: 2010/1.

\subsection{Protein isolation}

The tissue samples were homogenized using a 25-gauge, 2-mL syringe in 50-mM Tris, pH 8.0, containing $150-\mathrm{mM} \mathrm{NaCl}, 1 \% \mathrm{NP} 40$, and 0.1\% SDS and protease inhibitor (2× stock, 05,892 970001 , Roche). For approximately $1 \mathrm{mg}$ of tissue, $60-\mu \mathrm{L}$ lysis buffer was used. Homogenates were incubated on ice for 30 minutes by gently mixing twice. They were centrifuged at 13,000 rpm for 20 minutes at $4{ }^{\circ} \mathrm{C}$ and supernatants were collected. The soluble protein concentration was determined by a Bradford assay (Bradford Reagent, B6916, Sigma, St. Louis, MO, USA) and bovine serum albumin (Sigma, St. Louis, MO, USA) was used as a protein standard, and the supernatants were aliquoted and stored at $-80^{\circ} \mathrm{C}$.

\subsection{Western blot analysis}

From each sample, $10 \mu \mathrm{g}$ of protein was loaded into the gels for detection of PSD-95 and SYP, and for GEP, $40 \mu \mathrm{g}$ of protein was loaded into a separate set of gels. Samples of cohorts from each of the 6 groups were run in an $8 \%-10 \%$ resolving gel under denaturing

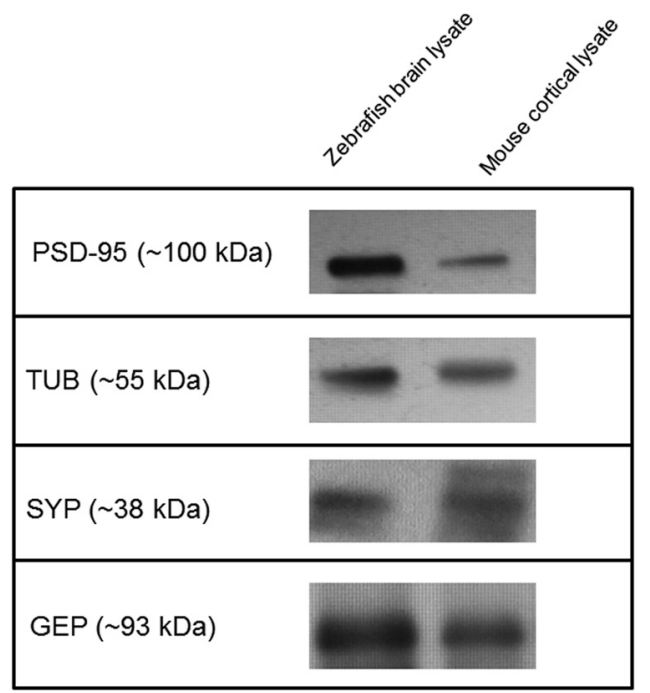

Fig. 1. Representative Western blots for the antibody validation experiments, which included mouse cortical lysate as a positive control. Postsynaptic density-95 (PSD-95), synaptophysin (SYP), gephyrin (GEP), and tubulin (TUB)

conditions. Following the gel separation, proteins were transferred onto PVDF membrane at $90 \mathrm{~V}$ for 90 minutes at $4{ }^{\circ} \mathrm{C}$. Membranes were blocked in $5 \%$ milk powder in tris-buffered saline with Tween 20 (20-mM Tris-HCl, $137-\mathrm{mM} \mathrm{NaCl}, 0.3 \%$ Tween 20, pH 7.6). Primary antibodies used for blotting were $\alpha$-SYP (Abcam, Cambridge, UK: ab32594, 1:20,000 dilution), $\alpha$-PSD-95 (Abcam, Cambridge, UK: ab18258, 1:5000 dilution), $\alpha$-GEP (Santa Cruz Biotechnology, Santa Cruz, CA, USA: sc-6411, 1:1000 dilution), and $\alpha-\beta$ tubulin (TUB; Cell Signaling Technology, Danvers, AM, USA: \#2146, 1:5000 dilution), which were used as a loading control. All antibodies were raised in rabbit except for GEP, which was raised in goat. The secondary antibodies used for blotting were antirabbit (Cell Signaling Technology, Danvers, AM, USA: \#7074, 1:2500 dilution) and antigoat (Abcam, Cambridge, UK: ab97100, 1:10,000 dilution) antibodies. All experiments were performed minimally in triplicates; therefore, for each animal sample, approximately 3 measurements were obtained. This was done to get an adequate representation of the brain level of these proteins. Femto Supersignal (Thermoscientific, Rockford, IL, USA: 34,095) was used for signal detection and membranes were exposed to photographic film. The films were developed and then photographed (Hyperprocessor, Amersham Life Sciences, UK). Quantitative analysis of the band densities was performed using the ImageJ program (NIH, Bethesda, MD, USA) and was done by author D. O. H., who was blind to the age and gender of the protein bands in the membrane. Thus, the quantification was performed in a completely unbiased manner. For the analysis of group differences, we chose 2 different normalization procedures. The first one was performed by normalizing each separate band representing a protein of interest, that is, PSD-95, to an average of the gel cohort for that protein of interest, that is, PSD-95. This normalization method has been previously used for quantifying age-related changes in synaptic proteins in rat hippocampus (Adams et al., 2008; Newton et al., 2008; Shi et al., 2007). This method allows for examining the effects of age and gender on all the synaptic proteins and the housekeeping protein of interest individually, as well as allowing for a comparison of their levels among them. In this paper, these values are designated as "normalized data." The other is the standard procedure of normalizing the intensity of each band that reflects the synaptic protein of interest, that is, PSD-95, to a corresponding intensity of the band of the housekeeping protein, that is, TUB, that was 


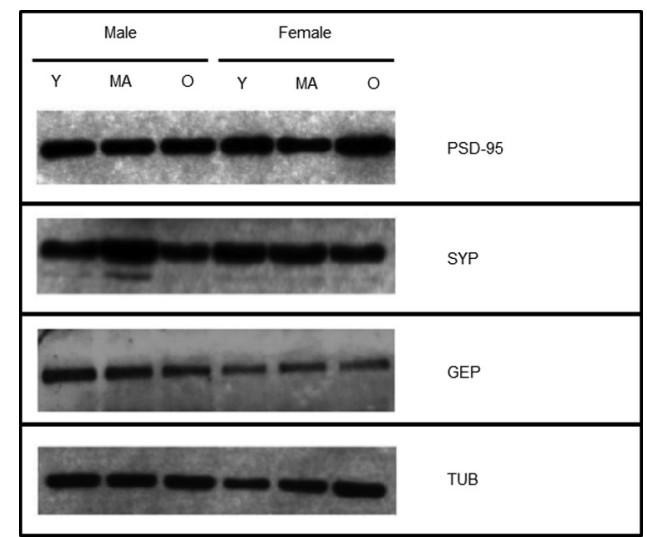

Fig. 2. Representative Western blots for synaptic proteins in the zebrafish brain across lifespan, postsynaptic density-95 (PSD-95), synaptophysin (SYP), gephyrin (GEP), and tubulin (TUB). Experimental groups were young (Y; 6-8 months), middle-aged (MA, 11-14.5 months), and old (0; 24-30 months).

predicted to not change with age or gender. This last procedure does not allow for determining whether there are specific effects of age and gender on the housekeeping protein of interest, that is, TUB. In this article, these values are designated as "TUB-normalized data." However, both procedures were performed to feel confident that synaptic protein differences were reflecting true biological changes among the groups. For both methods, all bands were normalized to the average intensity of the cohort within the membrane to control for possible variations across trials due to differences in exposure time. Moreover, as stated above, multiple measurements were taken per sample. Thus, any interassay differences would be minimized and not likely contribute to any biological differences among the groups.

\subsection{Statistical analysis}

Statistical analysis was performed on the band intensities obtained from the ImageJ program using the SPSS program (IBM, Istanbul, Turkey). Assumptions of a normal distribution and homogeneity of variance were tested with Kolmogorov-Smirnov and Levene tests, respectively, and in all cases these assumptions were fulfilled. Thus, a 2-way analysis of variance (ANOVA) with the factors of age with 3 levels (young, middle-aged and old) and gender with 2 levels (male and female) were employed to determine significant differences among groups. To validate these results, we also performed a multivariate analysis of variance (MANOVA) with the 2 factors, age and gender, on the dependent variables that showed significance in the 2-way ANOVA results. For the analyses of the very old females, a 1-way ANOVA with a factor of age with 4 levels (young, middle-aged, old, and very old) was conducted on the synaptic protein expression levels. To analyze region-specific alterations, a 3-way ANOVA with the factor of age with 2 levels (young and old), gender with 2 levels (female and male), and region with 3 levels (Tel, TeO, and cerebellum) was applied to the data from the microdissected zebrafish brains. Univariate tests were followed by post hoc analysis with a Bonferroni correction, which uses more stringent cutoffs for significance. To analyze the data for any potential significant interactions, a simple effects analysis was applied. The purpose of this analysis is to break the levels of an independent variable and show its effects on the levels of the other independent variable (Field, 2009). Significance levels in all cases were set at $p<0.05$.

Further investigation of the protein expression levels was performed using principal component analysis (PCA). These PCA tests were done to determine how the expression levels of these proteins were changing relative to each other but independent of the factors used in the previous ANOVA and MANOVA analysis. This analysis also indicated the correlations in these protein levels within the factors that were used. Doing this type of multivariate analysis further expands our understanding of how these proteins are changing relative to one another within groups. For component extraction, the eigenvalues were set above the 0.5 level.

\section{Results}

\subsection{Western blot analysis of synaptic proteins yields bands at the} expected molecular weights

A very common and formidable problem for investigators performing protein analyses in zebrafish tissues is the lack of working antibodies. Thus, in the present study initially 4 commercially available antibodies for Western blot analyses, which were not previously used in experiments involving zebrafish brains, have to be tested and optimized. The antibodies were chosen based on their sequence homology of the epitope and tested in our laboratory. Following many trials, which also included mouse cortex as a positive control, the optimal protein concentrations and antibody dilutions were determined such that the visualized protein bands were running at the expected molecular weights in both tissues (Fig. 1). In our Western blotting experiments, specific bands for our 3 synaptic proteins of interest and a reference protein were observed. Visualized bands for all proteins were observed at the expected molecular weights (Figs. 1 and 2). For $\alpha$-PSD-95, the molecular weight of the band was $100 \mathrm{kDa}$, for $\alpha$-SYP $38 \mathrm{kDa}$, for $\alpha$-GEP $93 \mathrm{kDa}$, and for TUB $55 \mathrm{kDa}$. All of these molecular weights are consistent with those reported in the literature for PSD-95 (Calkins et al., 2011), SYP (Riberio et al., 2014), GEP (Yang et al., 2013), and TUB (Lamming et al., 2014). To examine the quantitative differences in the synaptic protein levels, whole brain lysates from male and female brains from each age group were run in cohorts in the gel as depicted in Fig. 2 and all samples were minimally run in triplicates. On one membrane, $\alpha$ PSD-95, $\alpha$-SYP, and $\alpha$-TUB were blotted simultaneously, and on another $\alpha$-GEP and $\alpha$-TUB were blotted sequentially. Thus, in addition to the observed effects of aging that will be presented, we have optimized and tested 4 specific antibodies that will work for Western blotting analysis of the adult zebrafish brain, which is important for future experiments performed by researchers working with this animal model.

3.2. Quantitative analysis reveals that key excitatory and inhibitory postsynaptic proteins, as well as a presynaptic protein in male and female zebrafish brains are differentially affected by age and gender

\subsubsection{Postsynaptic density-95}

Postsynaptic density-95 is a clustering protein for NMDA and AMPA receptors and its levels alter excitatory synaptic functioning in the brain (Prange et al., 2004). Our quantitative analysis of both normalized data (Fig. 3A) and TUB-normalized data (Fig. 3B) revealed that in young animals, males had higher brain levels of PSD-95 as compared with females, and across lifespan, these levels increased in females but did not appear to change in males. The statistical analysis demonstrated no significant effect of age or gender but a significant age by gender interaction was observed $\left(F_{(2,30)}=5.43, p=0.010\right.$, Fig. $\left.3 \mathrm{~A}\right)$. This analysis indicates that the differences in the PSD-95 levels with age are dependent on gender. The simple effects analysis revealed that the effect of gender was significant in the young age groups $(p=0.011)$, and the effect of age was significant in females $(p=0.027$; Fig. $3 \mathrm{~A})$. In the 

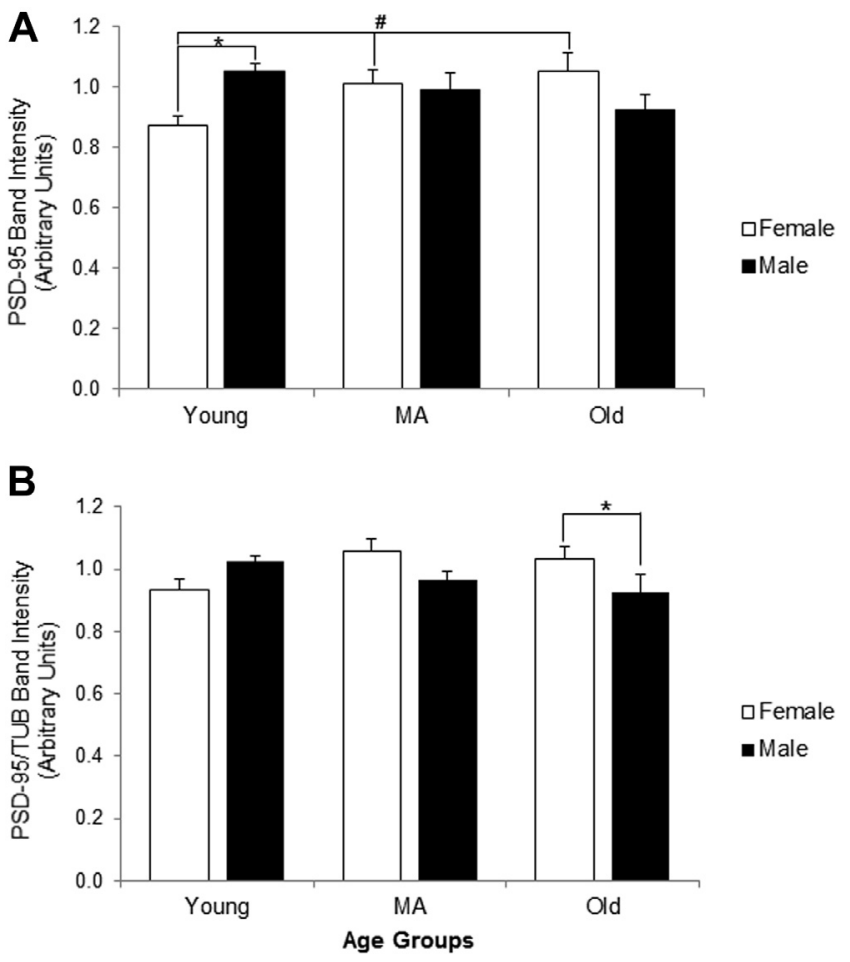

Fig. 3. Postsynaptic density-95 (PSD-95) levels increase in aged female zebrafish brains but are stable in males. Data are illustrated with both normalized PSD-95 levels (A) and TUB-normalized PSD-95 levels (B). Quantitative differences in PSD-95 levels according to age and gender are illustrated. The results from the experiments were performed in triplicates and the group means + standard error (SE) for males (black bars) and females (white bars) are represented. *Gender within age, ${ }^{*}$ Age within gender, All symbols $=p<0.05$. Abbreviation: MA, middle-aged.

TUB-normalized data, a significant age by gender interaction was also apparent in the PSD-95 expression levels $\left(\mathrm{F}_{(2,30)}=4.38, p=\right.$ 0.022 , Fig. $3 \mathrm{~B})$. The trend and alterations were the same as the normalized data, with the only difference being that the simple effects analyses of the TUB-normalized data demonstrated that the effect of gender was significant in the old group ( $p=0.049$ ), whereas in the normalized data the effect of age was marginally significant in the females $(p=0.059)$. These results suggest that in females this excitatory element of synaptic integrity increases across lifespan, whereas in males these levels are stable throughout the aging process, and the state of PSD-95 protein levels during brain aging is dependent on gender.

\subsubsection{Gephyrin}

Gephyrin is a clustering protein for both $\mathrm{GABA}_{\mathrm{A}}$ and glycine receptors (Chen et al., 2012; Dejanovic et al., 2014). Our quantitative analysis of normalized data seemed to indicate that across age GEP levels remained stable in females, but in middle-aged males it was increased as compared with young fish and then decreased in old animals back to the level of the young. However, no significant main effects of age and gender were observed $\left(F_{(5,30)}=2.012, p=0.106\right.$; Fig. $\left.4 \mathrm{~A}\right)$, although a marginally significant age by gender interaction was seen $\left(\mathrm{F}_{(2,30)}=2.840, p=0.074\right.$; Fig. 4A). The expression pattern of GEP was also similar in the TUB-normalized data with no significant main effect of age or gender $\left(F_{(5,30)}=1.766, p=0.150\right.$, Fig. $\left.4 B\right)$. Numerically, the pattern of differences in males from both data sets was similar, which suggests that there could be potential changes across lifespan in male zebrafish brains. In addition, there may be functional differences in the excitatory/inhibitory balance, since PSD-95 levels
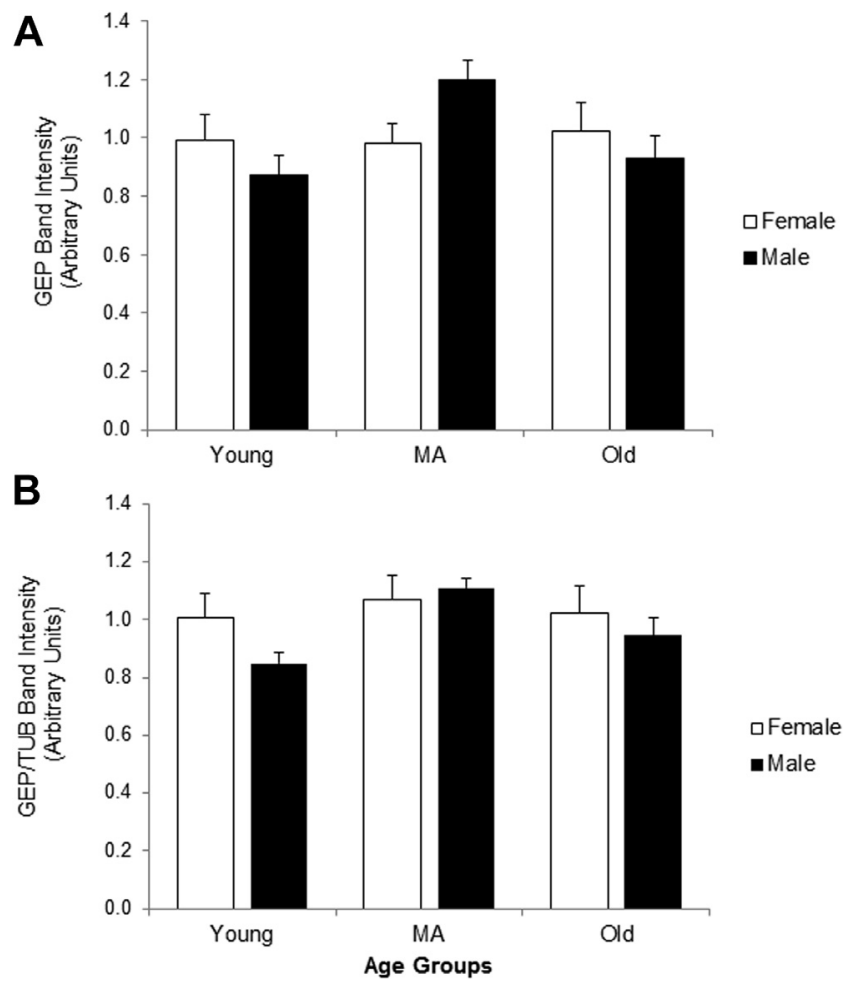

Fig. 4. Gephyrin (GEP) levels remain stable in male and female zebrafish brains across lifespan. Data are illustrated with both normalized GEP levels (A) and TUB-normalized GEP levels (B). Quantitative differences in gephyrin levels according to age and gender are illustrated. The results from the experiments were performed in triplicates and the group means + standard error (SE) for males (black bars) and females (white bars) are represented. Abbreviation: MA, middle-aged.

are changing relative to GEP. This could change the overall synaptic transmission and plasticity in the aged brain.

\subsubsection{Synaptophysin}

SYP is a presynaptic glycoprotein that is a $\mathrm{Ca}^{2+}$ sensor and regulates synaptic vesicle trafficking and endocytosis (Kwon and Chapman, 2011). Our quantitative analysis of normalized SYP protein levels demonstrated that middle-aged males had a higher level of this protein relative to females but in old animals, females had a higher level relative to males. Moreover, across lifespan SYP decreased in males and seemed to increase in females. Statistical analysis revealed that for SYP there was a significant age by gender interaction $\left(\mathrm{F}_{(2,29)}=8.006, p=0.002\right.$; Fig. 5A). The simple effects analysis indicated that there is a significant effect of the gender in both the middle-aged ( $p=0.004)$ and old groups $(p=0.017)$, and age has a significant effect in the male fish $(p=0.01)$. In the TUBnormalized data, a significant age by gender interaction was also shown $\left(F_{(2,29)}=4.469, p=0.020\right.$, Fig. $\left.5 B\right)$. Simple effects analyses demonstrated that the effect of gender was significant in the middle-aged group ( $p=0.019)$, and the effect of age was only significant in male group $(p=0.001)$. This pattern of results was the same as that shown in the normalized data analysis. Moreover, a significant main effect of age was observed in the TUB-normalized SYP levels $\left(\mathrm{F}_{(2,29)}=4.666, p=0.018\right.$, Fig. 5B), and multiple comparisons revealed that old males had significantly reduced levels of SYP as compared with the middle-aged males $(p=0.004)$. The results suggest that the level of this presynaptic protein, which is involved in presynaptic vesicle trafficking, is differentially affected by age in males and females. Thus, presynaptic release may be functioning differently in males and females and contribute to synaptic plasticity differences during the aging process. 

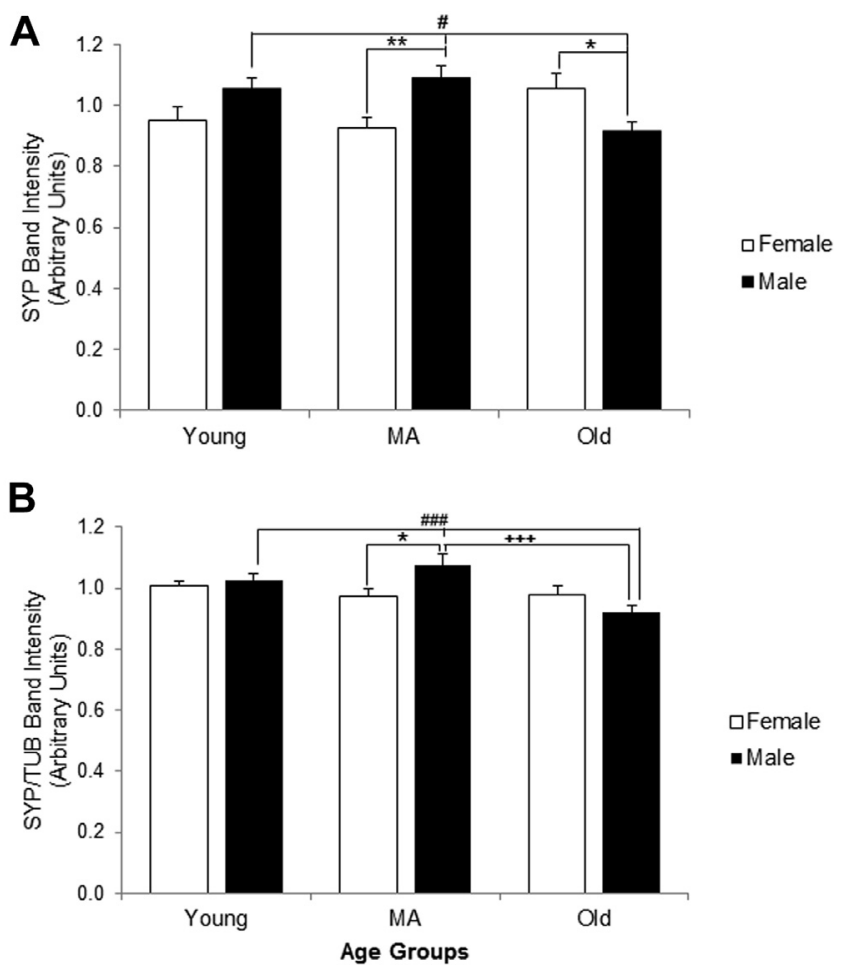

Fig. 5. Synaptophysin (SYP) levels decrease in old male zebrafish brains but remain stable in females. Data are illustrated with both normalized SYP levels (A) and TUBnormalized SYP levels (B). Quantitative differences in synaptophysin levels according to age and gender are illustrated. The results from the experiments were performed in triplicates and the group means + standard error (SE) for males (black bars) and females (white bars) are represented. ${ }^{*}$ Gender within age, ${ }^{\#}$ Age within gender, ${ }^{+}$Main effect, One symbol $=p<0.05$; Two symbols $=p<0.01$; Three symbols $=p<0.005$. Abbreviation: MA, middle-aged.

\subsubsection{Tubulin}

$\beta$-Tubulin is one of the cytoskeletal components that is generally used for the verification of equal protein loading (Lamming et al., 2014). TUB was quantified as a loading control only using the first normalization procedure and we observed no significant differences across the different ages or genders $\left(\mathrm{F}_{(5,30)}=1.616, p=0.186\right)$. The average TUB levels are reported in Table 1 along with the standard error of the mean. As was mentioned in the Methods section,

Table 1

Tubulin protein levels in male and female zebrafish brains

\begin{tabular}{llll}
\hline Group & $\mathrm{N}$ & Mean & Std. error \\
\hline Female-young & 6 & 0.96 & 0.029 \\
Male-young & 6 & 1.025 & 0.018 \\
Female-middle-aged & 6 & 0.946 & 0.036 \\
Male-middle-aged & 6 & 1.051 & 0.039 \\
Female-old & 6 & 1.020 & 0.036 \\
Male-old & 6 & 0.988 & 0.029 \\
Female-very old & 4 & 1.048 & 0.025 \\
Tel-female-young & 3 & 1.163 & 0.073 \\
Tel-male-young & 3 & 0.924 & 0.024 \\
Tel-female-old & 3 & 0.888 & 0.140 \\
Tel-male-old & 3 & 0.972 & 0.040 \\
TeO-female-young & 3 & 0.970 & 0.099 \\
TeO-male-young & 3 & 0.849 & 0.080 \\
TeO-female-old & 3 & 0.996 & 0.046 \\
TeO-male-old & 3 & 0.975 & 0.091 \\
Ce-female-young & 3 & 1.016 & 0.065 \\
Ce-male-young & 3 & 0.962 & 0.043 \\
Ce-female-old & 3 & 1.190 & 0.087 \\
Ce-male-old & 3 & 1.094 & 0.058 \\
\hline
\end{tabular}

Key: Ce, cerebellum/medulla/spinal cord; Tel, telencephalon; TeO, optic tectum. quantitative differences in TUB levels can only be observed by using the first normalization procedure, that is, normalizing the intensity of the band across the average of the gel cohort. By analyzing tubulin in the non-TUB normalized condition, that is, normalized data, we could determine if there were any direct effects of age and gender on TUB levels. For the second procedure, when we normalized each synaptic protein of interest to a tubulin value, we needed to assume that tubulin levels were not changing. In the TUB-normalized condition, a change in tubulin levels on their own may alter the outcome of the synaptic results such as that of PSD-95, for example. These data support the use of this protein as a housekeeping protein for both normalized and TUB-normalized data comparisons because it is not changing significantly with age or gender. In addition, it indicates that there are no significant loading or pipetting errors in our experimental setup.

\subsection{Validation of the effects of age and gender on key synaptic protein expression levels in males and females across lifespan}

As was mentioned in the Methods section, we followed up the 2-way ANOVA results using MANOVA. We observed similar patterns in the effects of age and gender on key synaptic protein expression levels, with the MANOVA demonstrating a significant age by gender interaction on the variables as a whole $\left(F_{(6,54)}=4.850, p=0.001\right.$; Wilk's $\left.\Lambda=0.422\right)$. When we examined the protein results individually, similar to the ANOVA results, we found significant age by gender interactions for the protein levels of PSD-95 ( $p=0.004)$ and SYP $(p=0.002)$, and marginally significant for $\operatorname{GEP}(p=0.055)$. These results validate the significant effects that we observed with the ANOVA statistics.

Since the presence of an age by gender interaction also was suggested by MANOVA, we performed a PCA to explore the variation of expression across all 3 synaptic proteins. A significant correlation was found between the protein levels of PSD-95 and SYP $(\mathrm{r}=0.57, p<0.0005)$, as well as SYP and GEP $(\mathrm{r}=0.42, p=0.007)$, whereas the correlation between PSD-95 and GEP was not significant. Visualization of samples in 2-dimensions using PC1 and PC2 accounted for $87.5 \%$ of the variation in synaptic protein expression (Fig. 6A). Due to an age by gender interaction, we also replotted samples within each age group separately demonstrating the relatively distinct age-specific distribution of males and females (Fig. 6B-D).

In the PCA using the complete dataset, the PC1 score explained most of the variation by referring to the magnitude of the overall change in the data and indicated that as the expression of these 3 proteins increased, so did the PC1 score of the sample (Fig. 6A). Considering all samples, the results demonstrated that that the protein levels of PSD-95 and SYP were highly correlated and this would suggest that SYP and PSD-95 were mainly driving the PC1 score but the GEP protein levels, which positively correlated with SYP, could be contributing to the significant effects (component loading $>0.5$ ). On the other hand, the PC2 score that was orthogonal to the PC1, denoted a significant portion of the variation that was due to GEP only and it was negatively correlated with PSD-95. This kind of relationship indicates that PC2 might reflect the inhibitory contributions to the excitatory-inhibitory balance, whereas PC1 is reflecting a more general sense of the synaptic integrity.

Aging has a complex pattern based on the components, thus, we also plotted samples in an age-specific manner. In PC1 of the young dataset, the effect of GEP was less than that observed in the total suggesting that PSD-95 and SYP explain most of the variation in the expression differences of young animals. Moreover, PC2 is mainly driven by GEP (Fig. 6B). A similar pattern was observed in the old group, with PSD-95, SYP, and GEP steering PC1, and PC2 being mostly correlated with GEP (Fig. 6D). Middle-aged animals had a 


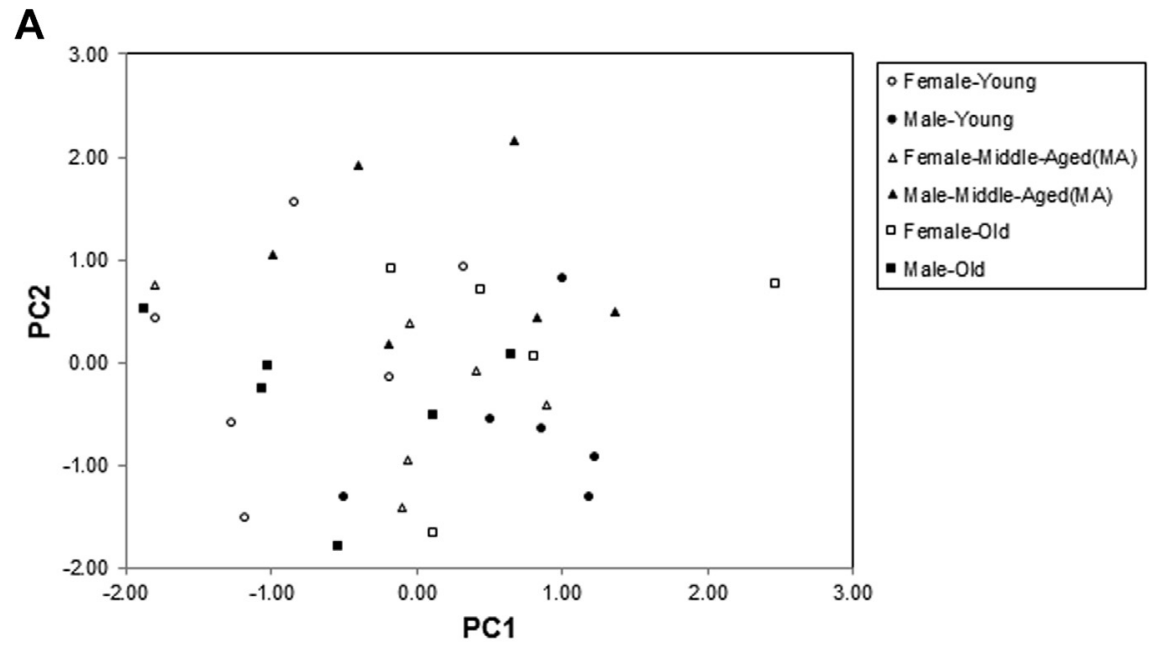

B

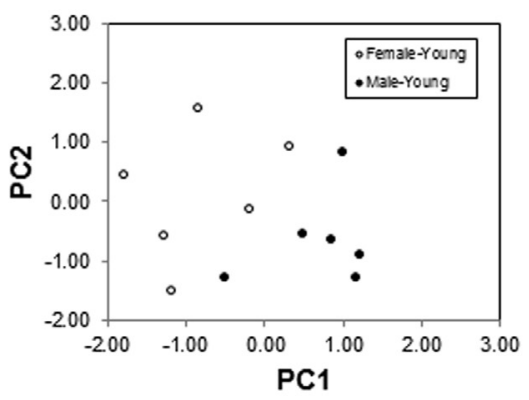

C

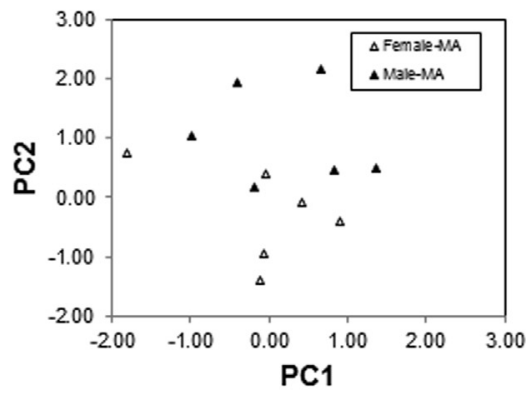

D

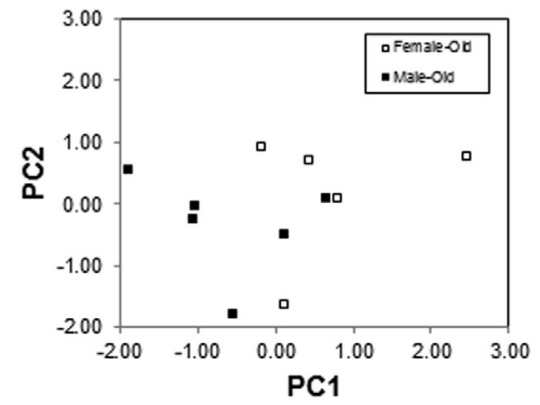

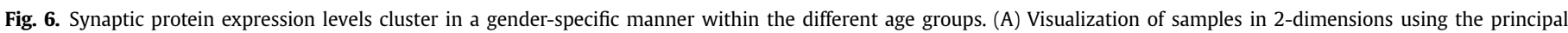

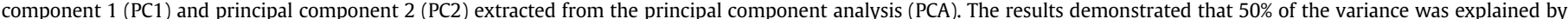

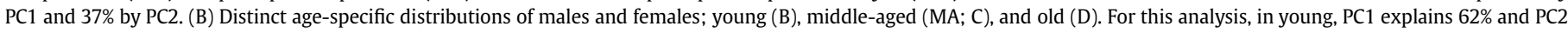
$36 \%$ of the variance; in MA, PC1 50\% and PC2 35\%; and in old, PC1 72\% and PC2 22\%. Groups are denoted by different symbols and represented in the legend.

relatively different clustering profile from the rest of the age groups such that the correlation between PSD-95 and SYP, which drives PC1 in the other groups, became weaker, and the correlation between SYP and GEP became stronger, with PSD-95 controlling the variation in PC2 (Fig. 6C). This kind of complex pattern might emphasize the importance of middle-aged groups in large-scale lifespan aging studies. Based on our data, alterations in the clustering profile of the protein expression levels indicate that middle-age might serve as a critical point where subtle age-related alterations begin. These data suggest that synaptic protein loss within different age groups is dependent on gender and that presynaptic and postsynaptic protein levels are correlated but excitatory and inhibitory proteins are not associated. Since our youngest age group is considered as young adult, because they have already reached sexual maturity (Kimmel et al., 1995; Parichy et al., 2009; Singleman and Holtzman, 2014), and they were compared to animals that are older and very old, these synaptic protein differences represent the effects of normal aging and not a maturation or developmental process.

\subsection{Synaptic proteins are stable in female brains even in} very old age

Since a pattern of increasing PSD-95 and SYP protein levels was observed in the old female brains, we determined if this change continued into very old age. On average, zebrafish can live up to 3-5 years (Kishi et al., 2009); so, we sampled brains from females that were going toward the end of their lifespan. Therefore, we added an additional set of 4 very old females (34-43 month old), and compared their key synaptic protein levels with the other females from the different aged groups that had been examined (Fig. 7). Using a 1-way ANOVA, our analysis revealed that there were no significant decreases or increases in synaptic proteins from the female brains even into very old age. This was true for PSD-95 protein levels $\left(\mathrm{F}_{(3,18)}=2.831, p=0.068\right.$; Fig. 7A), for GEP protein levels $\left(\mathrm{F}_{(3,17)}=0.045, p=0.987\right.$; Fig. $\left.7 \mathrm{C}\right)$, and for SYP protein levels $\left(F_{(3,17)}=1.389, p=0.280\right.$; Fig. $\left.7 \mathrm{E}\right)$. Moreover, no significant effects were observed in the TUB levels $\left(\mathrm{F}_{(3,18)}=0.903, p=0.165\right.$; Table 1$)$. Additional analyses using TUB-normalized values demonstrated the same pattern of results for PSD-95 $\left(\mathrm{F}_{(3,18)}=2.400, p=0.102\right.$; Fig. 7B), GEP $\left(\mathrm{F}_{(3,17)}=0.284, p=0.836\right.$; Fig. 7D), and SYP $\left(F_{(3,17)}=1.364, p=0.287\right.$; Fig. $\left.7 F\right)$. These results suggest that the levels of these key synaptic proteins tend to be stable and preserved in the female brains even at very old age.

\subsection{Region-specific alterations in key synaptic proteins during aging}

To examine the possibility of region-specific alterations in the zebrafish brain, whole brain tissue was microdissected into 3 parts including the Tel, TeO, and Ce. For both normalized and TUBnormalized values of PSD-95, no significant age or gender effects were observed in any microdissected regions $\left(\mathrm{F}_{(11,24)}=2.173, p=\right.$ 0.054 ; Fig. $8 \mathrm{~A} ; \mathrm{F}_{(11,24)}=1.086, p=0.412$; Fig. $\left.8 \mathrm{~B}\right)$. Similarly, for GEP 
A

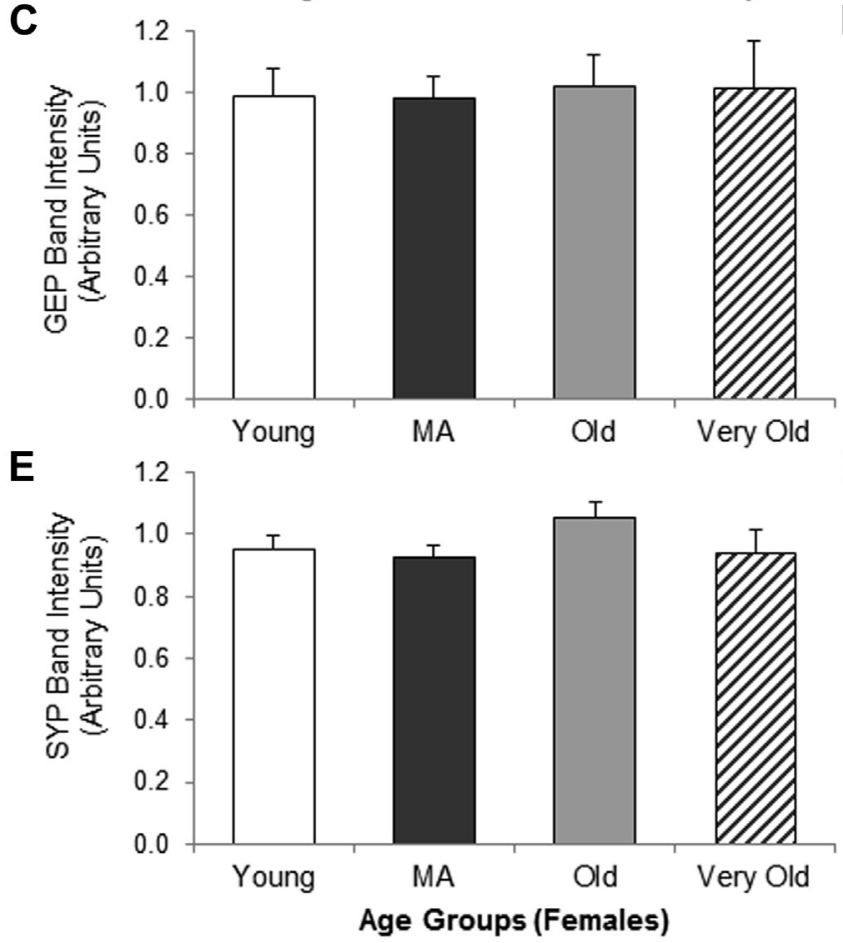

B

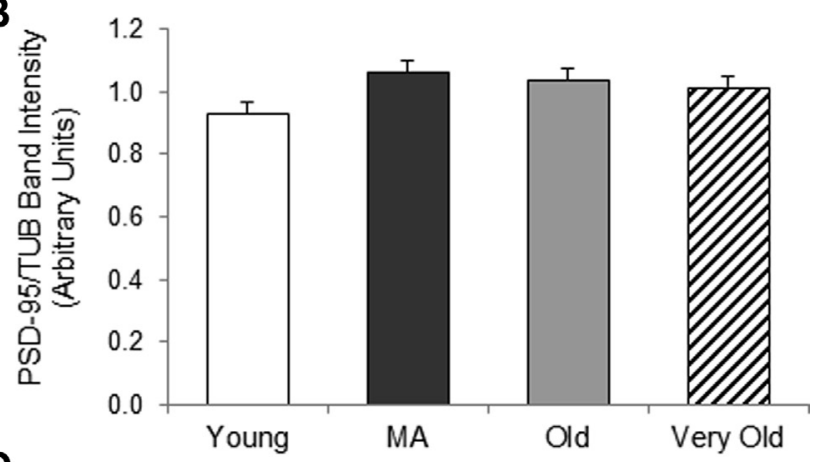

D

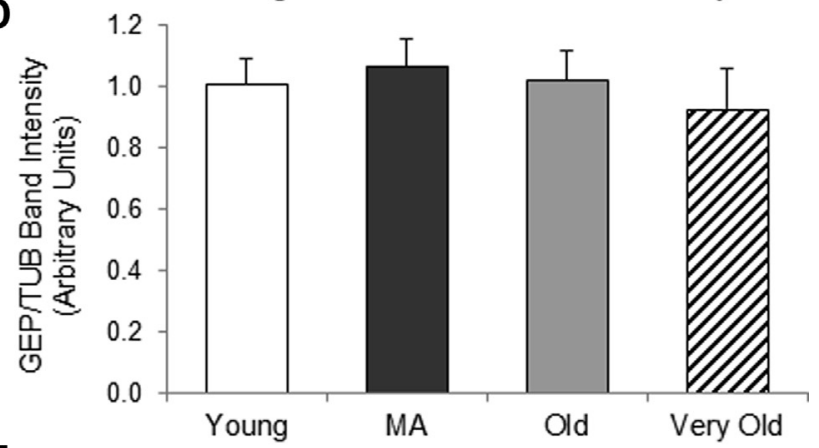

$\mathbf{F}$

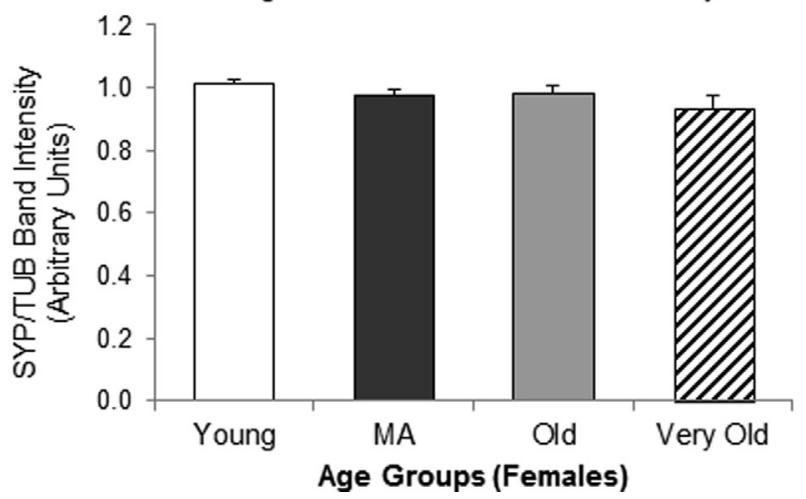

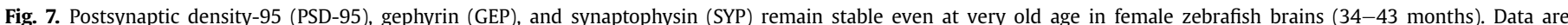

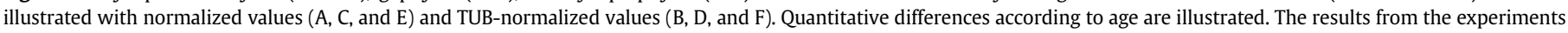
were performed in triplicates and the group means + standard error (SE) are represented (bars). Abbreviation: MA, middle-aged.

no significant differences were seen among any of the ages or genders $\left(\mathrm{F}_{(11,24)}=0.224, p=0.993\right.$; Fig. $8 \mathrm{C} ; \mathrm{F}_{(11,23)}=0.656, p=$ 0.763; Fig. 8D). Finally, for SYP there were no significant differences found in young and old males and females $\left(\mathrm{F}_{(11,24)}=0.513, p=\right.$ 0.876; Fig. 8E; $\mathrm{F}_{(11,24)}=1.015, p=0.463$; Fig. $\left.8 \mathrm{~F}\right)$. The reported degrees of freedom reflect the corrected model rather than individual comparisons since there were no significant main effects or interactions of age and gender. Interestingly, we did notice some similar patterns of numerical differences between the regionspecific and whole brain analyses. For example, in the Tel, changes in PSD-95 protein expression levels reflect a pattern similar to that of the whole zebrafish brain analyses with old females having higher levels of this synaptic protein than old males (Figs. 3A, B and 8A, B). Moreover, for SYP protein levels, the data analysis of the Tel also reflect an analogous relationship to the whole zebrafish brain analyses, with old females having higher levels than old males (Figs. 5A, B and 8E, F). Since the whole brain changes that we found appear to parallel the differences in the Tel more than the other regions, it would be logical to assume that any effects of age and gender on synaptic protein levels observed using whole brain samples are being driven by alterations specifically in this area. This is interesting because proportionally this area represents approximately $10 \%-15 \%$ of the total brain weight. Thus, a small area has a significant contribution on the overall alterations in the aging zebrafish brain. Moreover, it may represent a region that is selectively vulnerable to the effects of aging as this portion of the zebrafish brain contains structures homologous to the hippocampus, amygdala, and the cortex, which are possibly more affected by the aging process (Almaguer et al., 2002; For review see; Morrison and Baxter, 2012).

\section{Discussion}

The present study demonstrated that the levels of PSD-95, a postsynaptic protein component of excitatory synapses, GEP, a postsynaptic protein component of inhibitory synapses, and SYP, a presynaptic marker, all of which are indicators of synaptic integrity, are relatively stable throughout the aging process. However, there are significant age by gender interactions observed for PSD-95 and SYP whole brain levels suggesting that the pattern of aging differs between male and female groups. In female zebrafish brains, PSD95 levels are preserved and increased in older ages, whereas, there 
A

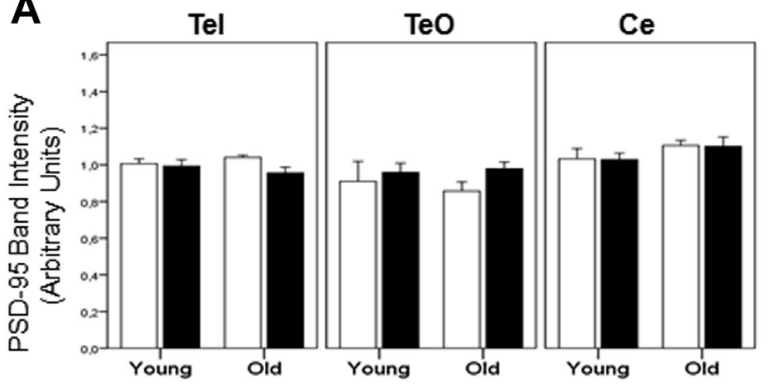

C

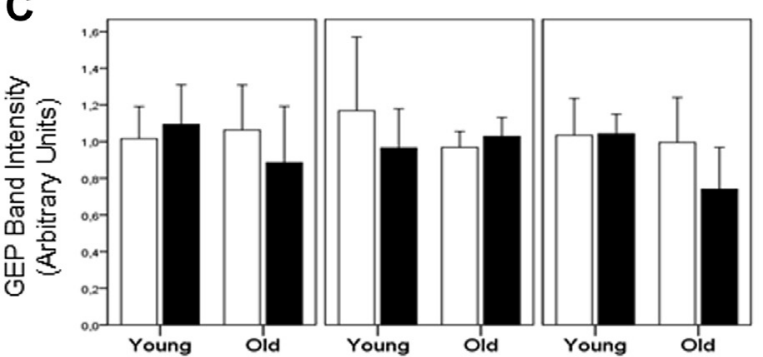

$\mathbf{E}$

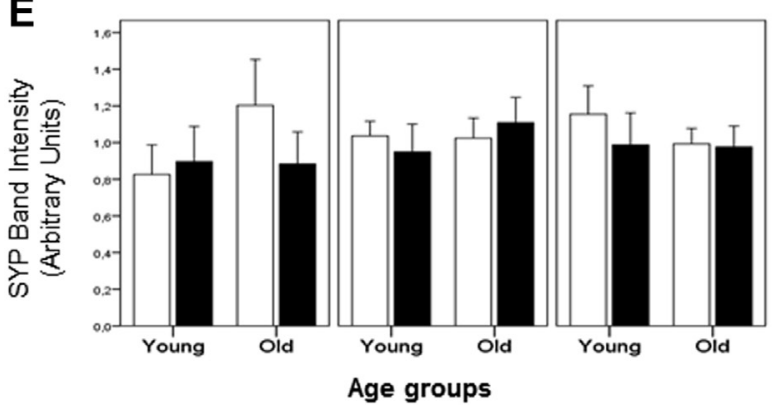

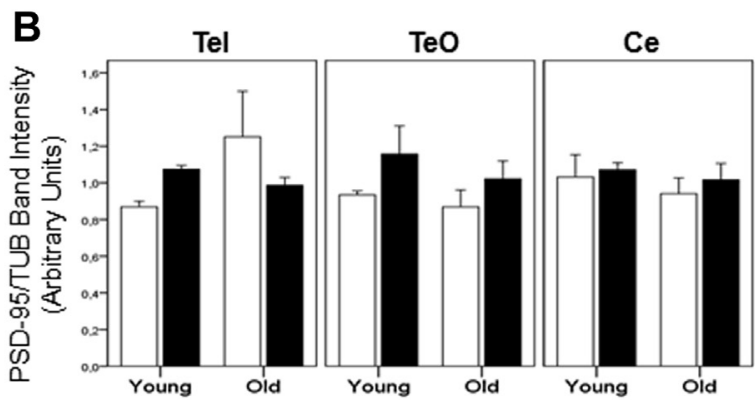
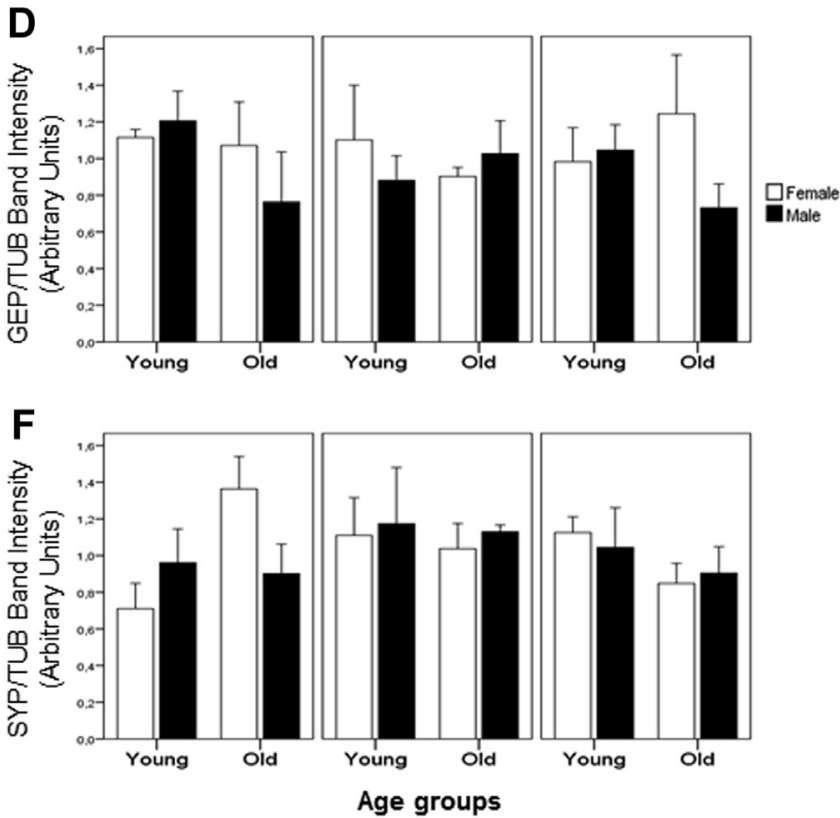

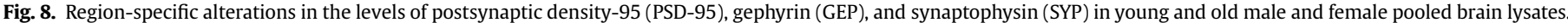

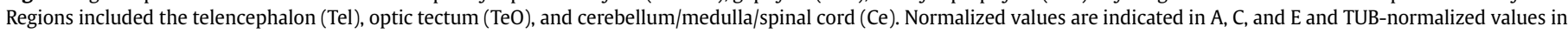
$\mathrm{B}, \mathrm{D}$, and $\mathrm{F}$. The results from the experiments were performed in triplicates and the group means + standard error (SE) are represented (bars).

is no evident alteration in the males throughout the aging process. A similar pattern was observed in the SYP levels; however, in females the levels of SYP seem to be stable throughout the lifespan but in the male group there is decrease during old age. These changes are occurring in the absence of any significant alterations in the GEP protein levels, although the levels seem to increase in the middle-aged males relative to young and old. Also, region-specific analysis indicates that these age-related differences may be driven by changes that are specific to the Tel, which is the region of the zebrafish brain involved in higher cognitive processing. Taken together, these data indicate that aging subtly alters the molecular dynamics of synapses in a sexually dimorphic manner.

While the zebrafish is becoming an increasingly popular model organism to study the effects of age and aging-associated disorders, to date little is known about the normal course of brain aging in this animal. Therefore, the first purpose of this study was to examine in zebrafish the effects of age, from young adulthood until very old, and gender on the levels of key synaptic proteins that are indicators of excitatory and inhibitory postsynaptic integrity and function. PSD-95, which is a scaffolding protein for NMDA and AMPA receptors, was used to estimate the excitatory components of synaptic integrity, and GEP, which is a scaffolding protein for $G_{A B A}$ and glycine receptors, was used to examine the inhibitory aspect of synaptic integrity. Since, significant interactions were observed in PSD-95 levels, and no significant alteration was observed in GEP levels, the excitatory/inhibitory balance is likely altered throughout the aging process and this occurs in a sexually dimorphic pattern. To illustrate, in aging female animals this balance could be shifted toward heightened excitation because of the increased levels of PSD-95 occurring without a change in GEP, as compared with the males. In previous studies, these 2 markers of synaptic function were examined in aging subjects. Results indicated that there is an age-related decrease in PSD-95 levels in the hippocampus of male rats (VanGuilder et al., 2011) and alterations in GEP levels in aged mice were dependent on cognitive status and the brain area studied (Majdi et al., 2009). Moreover, it was shown that the excitatory/ inhibitory ratio derived from the levels of these 2 excitatory and inhibitory scaffolding proteins were changing in the aged groups (Majdi et al., 2007). Based on these results, it is reasonable to conclude that the excitatory/inhibitory balance could be different in aged females as compared with the male animals. Future studies will be aimed at examining the functional consequences of these variations in zebrafish brains.

One reason that we did not observe any significant differences in GEP levels or larger differences in PSD-95 in the first set of experiments could be due to the fact that the expression levels of these markers are different throughout the various brain regions (Majdi et al., 2009; Tyagarajan and Fritschy, 2014; VanGuilder et al., 2011). The studies focusing on age-related changes in GEP levels (Majdi et al., 2009), for example, examined the protein levels in a region-specific manner in the brains of the mice. To determine whether there are regional differences in the protein levels, we 
microdissected the zebrafish brain into 3 separate regions: (1) Tel; (2) TeO; and (3) Ce. While this procedure increased the anatomic specificity, due to the small of the size of the zebrafish brain regions, specifically the Tel, the tissue samples needed to be pooled to obtain adequate amounts of protein for the Western blot analysis. Thus, we used region-specific brain samples pooled from 3 subjects for each young and old male and female group to determine if there were any significant differences among the brain areas. Interestingly, the results from this experiment indicated that similar to the whole brain analysis, the levels of these proteins are relatively stable. However, there were numerical differences in PSD-95 and SYP levels in the Tel, not observed in any other region, that show a comparable pattern similar to the whole brain. Therefore, our data analysis of the changes in synaptic protein levels indicated that those observed in the whole brain parallel the same direction of those in the Tel more than any other region. Interestingly, this area only represents approximately $10 \%-15 \%$ of the total brain weight, and while being small in nature has a significant contribution to the overall alterations in the aging zebrafish brain. Thus, the differences in the whole brain are likely being driven by changes in this region. Since the Tel contains structures homologous to the hippocampus, amygdala, and the cortex, it may be more vulnerable to the effects of aging (Almaguer et al., 2002; For review see; Morrison and Baxter, 2012).

While we expected to observe significant differences between young and old animals by using a region-specific dissection, as had been done by other groups (Adams et al., 2008; Newton et al., 2008; Shi et al., 2007), this was not the case. We believe that one reason for this is that since the brain samples needed to be pooled for each age group to obtain adequate protein concentrations, there was a greater amount of variance that was not observed when using large numbers of individual samples and this increased variability could have blurred the results. In addition, analyzing proteins with regionspecific microdissection does not give information about specific subregion changes that might be occurring across specific lamina in the zebrafish brain. To answer this question, a comprehensive quantitative analysis using immunohistochemical techniques needs to be performed. Currently, the antibodies used do not work adequately to perform this analysis and this is an overarching problem for using zebrafish in these types of experiments. Thus, we are diligently working on trying to optimize them, as well as develop new antibodies, and then such experiments will be performed.

Another reason for the difference between our results and previously reported studies could be the distribution of the old population. In the previous studies using rats as the model organism, old animals tended to be divided into unimpaired versus impaired groups with respect to their cognitive performance, and more pronounced differences in terms of protein expression pattern were observed between the young groups and old cognitively impaired groups (Majdi et al., 2007, 2009; VanGuilder et al., 2011). In our case, the old group was not segregated with respect to cognitive performance, and, therefore, since it likely included both unimpaired and impaired animals, any striking protein differences might have been blurred. Thus, future studies will be aimed at examining protein levels with relationship to cognitive status during aging, which has been shown to decline in zebrafish (Yu et al., 2006). Finally, while there was no significant change in GEP levels, we did observe a marginally significant difference in males such that middle-aged animals have higher GEP protein levels than young or old. These data are consistent with that reported by Pinto et al. (2015) and also support the idea that there could be age-related differences in the excitatory/ inhibitory balance.

Another purpose of this study was to examine a presynaptic marker in the aging zebrafish brain, in addition to the postsynaptic markers of excitatory and inhibitory synapses. This was done to have information about possible age-related alterations in presynaptic function. We chose the presynaptic protein, SYP, which has potential roles in vesicle trafficking and recycling (Kwon and Chapman, 2011). Previous studies have indicated that there are age-related decreases in SYP levels in the hippocampus of male mice (Adams et al., 2008; Saito et al., 1994; Smith et al., 2000), and our results indicated that there is a significant gender by age interaction in SYP levels. Thus, in the males SYP levels are reduced at old age when they were compared with the females and this would be consistent with the previously reported data. These decreases in SYP levels would likely indicate that vesicle trafficking and recycling are possibly altered in the aging zebrafish brain. However, it might also be the case that changes in SYP levels reflect differences in synapse size or number. We are currently investigating all of these possibilities.

This study was designed to help further expand our knowledge about the aging zebrafish brain since it is becoming a popular model for studying brain aging and age-related diseases, and our data taken together with other studies from the literature are integral for this process. Converging lines of evidence have demonstrated that this model resembles mammalian aging models in many aspects. A previous study has shown that SA-ß-gal activity and oxidized proteins accumulate in the muscle tissues of aged zebrafish groups as compared with young controls, and also gradual senescence in the regenerative and reproductive abilities of the aged zebrafish have been reported (Kishi et al., 2003). Moreover, we reported that $S A-\beta$-gal also accumulated in the aged zebrafish brain (Arslan-Ergul et al., 2016). These results are in parallel to the mammalian aging models (Arslan-Ergul et al., 2016; Kishi et al., 2003). Age-related loss of telomerase function, which results in shortened telomere lengths, has also been observed in zebrafish (Anchelin et al., 2013; Arslan-Ergul et al., 2016). Moreover, as related to telomere length, zebrafish show high resemblance to humans, whereas, there is a strong difference between humans and mice in terms of this marker. In terms of age-associated accumulation of oxidized proteins and lipofuscin deposits, although zebrafish have high antioxidant activities compared with mammals, in the specific telencephalic areas of old zebrafish, significant increases in oxidized proteins and lipofuscin deposits were observed and they are associated with spatial and associated learning deficits in old zebrafish (Ruhl et al., 2015). Therefore, it can be concluded that the response against oxidative stress shows a remarkable resemblance between mammals and zebrafish. Moreover, the general markers of aging in humans and mammals are also observed in zebrafish; therefore, it definitely represents a promising model for studying human aging.

The most interesting finding of the current study is the sexually dimorphic pattern in the subtle age-related molecular alterations in synaptic protein levels. There are very few studies conducted using the zebrafish model to determine whether or not there are genderrelated patterns that are parallel to studies using mammalian models. Reports from laboratories using zebrafish as a model organism for studying age-related brain changes are consistently beginning to indicate that there are many differentially expressed transcripts and genes between male and female brains (Arslan-Ergul and Adams, 2014; Toyama et al., 2009). Parallel to those results, human studies conducted on tissues from intact individuals of different age ranges indicate that during the aging process different categories of genes are predominantly altered in males and females (Berchtold et al., 2008). In addition to these differences in gene expression patterns, neurogenic capabilities of male and female brains vary in a gender-dependent manner and this disparity could have an effect on the course of normal aging. In mammalian studies, data indicate that sex-specific neurogenesis 
occurs in the hippocampal areas (Zhang et al., 2008). Moreover, studies conducted in adult zebrafish showed parallel results (Ampatzis and Dermon, 2007; Ampatzis et al., 2012). For example, in the medial zone of the dorsal Tel, the periventricular nucleus of the posterior tuberculum, and the ventral portion of the periventricular pretectal nucleus, which are all part of the forebrain, there are higher levels of cycling cells in female animals, and in the dorsal zone of periventricular zones there are higher levels of cycling cells in males (Ampatzis et al., 2012). In addition, in the cerebellum of the zebrafish a similar kind of dimorphism was observed between males and females in terms of neurogenic capabilities (Ampatzis and Dermon, 2007). Besides those reports, unfortunately, gender differences and related patterns tend to be underestimated in many aging studies, and generally experimental designs are solely made with male animals. Therefore, sexually dimorphic patterns at the level of the expression of key synaptic proteins observed in the current study will be really promising for the further studies and experimental designs.

\section{Conclusion}

In conclusion, the results of this study indicate that the effect of aging, from young adult to very old, on synaptic protein levels that are markers of synaptic function and integrity of excitatory and inhibitory synapses depends on the gender of zebrafish. Moreover, the protein levels of PSD-95, the NMDA and AMPA receptorclustering protein at excitatory synapses, and the presynaptic protein, SYP, which is important for vesicle recycling and trafficking are changing with increasing age, but GEP, the $\mathrm{GABA}_{\mathrm{A}}$ and glycine receptor clustering protein at inhibitory synapses, is relatively stable. Our data suggest that the brains of aged female zebrafish, as have been seen in mammals, may shift toward heightened excitation but males do not show this similar pattern. Moreover, if there is a shift toward higher excitation in female brains, this would be consistent with other studies demonstrating that females may be more vulnerable to excitotoxic injury with increasing age (Adams et al., 2001; Auer, 1996). Thus, age differentially affects the molecular dynamics of synapses in zebrafish and examining both genders in relationship to this, as is done in other species, will be important for our complete understanding of normal brain aging. These data provide an important baseline for the neurobiological changes that are occurring in this model organism, and are a promising start to helping establish the zebrafish as a model for human brain aging and revealing the effects of sexual dimorphism on age-related synaptic alterations.

\section{Disclosure statement}

The authors have no actual or potential conflicts of interests.

\section{Acknowledgements}

This work was supported by a European Molecular Biology Organization (EMBO) Installation Grant to Michelle M. Adams.

The authors would like to thank Tulay Arayici for excellent technical assistance with the experiments.

\section{References}

Adams, M.M., Shah, R.A., William, G.M.J., Morrison, J.H., 2001. Different modes of hippocampal plasticity in response to estrogen in young and aged female rats. Proc. Natl. Acad. Sci. U. S. A. 98, 8071-8076.

Adams, M.M., Shi, L., Linville, M.C., Forbes, M.E., Long, A.B., Bennett, C., BrunsoBechtold, J.K., 2008. Caloric restriction and age affect synaptic proteins in hippocampal CA3 and spatial learning ability. Exp. Neurol. 211, 141-149.
Almaguer, W., Estupiñán, B., Uwe Frey, J., Bergado, J.A., 2002. Aging impairs amygdala-hippocampus interactions involved in hippocampal LTP. Neurobiol. Aging 23, 319-324.

Ampatzis, K., Dermon, C., 2007. Sex differences in adult cell proliferation within the zebrafish (Danio rerio) cerebellum. Eur. J. Neurosci. 25, 1030-1040.

Ampatzis, K., Makantasi, P., Dermon, C., 2012. Cell proliferation pattern in adult zebrafish forebrain is sexually dimorphic. Neuroscience 226, 367-381.

Anchelin, M., Alcaraz-Pérez, F., Martínez, C., Bernabé-García, M., Cayuela, M. Mulero, V., 2013. Premature aging in telomerase-deficient zebrafish. Dis. Models Mech. 6, 1101-1112.

Arslan-Ergul, A., Adams, M.M., 2014. Gene expression changes in aging Zebrafish (Danio rerio) brains are sexually dimorphic. BMC Neurosci. 15, 29.

Arslan-Ergul, A., Erbaba, B., Karoglu, E.T., Halim, D.O., Adams, M.M., 2016. Shortterm dietary restriction in old zebrafish changes cell senescence mechanisms. Neuroscience 334, 64-75.

Auer, R., 1996. Effect of age and sex on N-methyl-D-aspartate antagonist-induced neuronal necrosis in rats. Stroke 27, 743-746.

Berchtold, N.C., Cribbs, D.H., Coleman, P.D., Rogers, J., Head, E., Kim, R., Cotman, C.W., 2008. Gene expression changes in the course of normal brain aging are sexually dimorphic. Proc. Natl. Acad. Sci. U. S. A. 105, 15605-15610.

Brandt, N., Vierk, R., Rune, G., 2013. Sexual dimorphism in estrogen-induced synaptogenesis in the adult hippocampus. Int. J. Dev. Biol. 57, 351-356.

Brody, H., 1955. Organization of cerebral cortex. III. A study of ageing in the human cerebral cortex. J. Comp. Neurol. 102, 511-556.

Calhoun, M., Kurth, D., Phinney, A., Jucker, M., Long, J., Hengemihle, J., Ingram, D., 1998. Hippocampal neuron and synaptophysin-positive bouton number in aging C57BL/6 mice. Neurobiol. Aging 19, 599-606.

Calkins, M., Manczak, M., Mao, P., Shirendeb, U., Reddy, P., 2011. Impaired mitochondrial biogenesis, defective axonal transport of mitochondria, abnormal mitochondrial dynamics and synaptic degeneration in a mouse model of Alzheimer's disease. Hum. Mol. Genet. 20, 4515-4529.

Chen, J., Villa, K., Cha, J., So, P., Kubota, Y., Nedivi, E., 2012. Article: Clustered dynamics of inhibitory synapses and dendritic spines in the adult neocortex. Neuron 74, 361-373.

Colon, E., 1972. The elderly brain. A quantitative analysis of the cerebral cortex in two cases. Psychiatr. Neurol. Neurochir. 75, 261-270.

Dayan, A., 1970. Quantitative histological studies on the aged human brain. Acta Neuropathol. 16, 85-94.

Dejanovic, B., Ebert, S., Neuser, F., Schwarz, G., Semtner, M., Meier, J., Lüscher, B. 2014. Palmitoylation of gephyrin controls receptor clustering and plasticity of GABAergic synapses. PLoS Biol. 12, 1-16.

Edelmann, K., Glashauser, L., Sprungala, S., Hesl, B., Fritschle, M., Ninkovic, J. Chapouton, P., 2013. Increased radial glia quiescence, decreased reactivation upon injury and unaltered neuroblast behavior underlie decreased neurogenesis in the aging zebrafish telencephalon. J. Comp. Neurol. 521, 3099-3115.

Field, A.P., 2009. Discovering Statistics Using SPSS. SAGE Publications, Los Angeles.

Friedrich, R.W., Jacobson, G.A., Zhu, P., 2010. Review: circuit neuroscience in zebrafish. Curr. Biol. 20, R371-R381.

Ganeshina, O., Berry, R., Nicholson, D., Geinisman, Y., Petralia, R., 2004. Differences in the expression of AMPA and NMDA receptors between axospinous perforated and nonperforated synapses are related to the configuration and size of postsynaptic densities. J. Comp. Neurol. 468, 86-95.

Ganz, J., Kroehne, V., Freudenreich, D., Machate, A., Geffarth, M., Braasch, I., Brand, M., 2014. Subdivisions of the adult zebrafish pallium based on molecular marker analysis. F1000research 3, 308.

Jahn, R., Schiebler, W., Ouimet, C., Greengard, P., 1985. A 38,000-dalton membrane protein (p38) present in synaptic vesicles. Proc. Natl. Acad. Sci. U. S. A. 82 , 4137-4141.

Kimmel, C.B., Ballard, W.W., Kimmel, S.R., Ullmann, B., Schilling, T.F., 1995. Stages of embryonic development of the zebrafish. Developmental Dyn. 203, 253-310.

Kishi, S., Slack, B.E., Uchiyama, J., Zhdanova, I.V., 2009. Zebrafish as a genetic model in biological and behavioral gerontology: where development meets aging in vertebrates - a mini-review. Gerontology 55, 430-441.

Kishi, S., Uchiyama, J., Baughman, A.M., Goto, T., Lin, M.C., Tsai, S.B., 2003. The zebrafish as a vertebrate model of functional aging and very gradual senescence. Exp. Gerontol. 38, 777-786.

Kneussel, M., Brandstatter, J., Laube, B., Stahl, S., Muller, U., Betz, H., 1999. Loss of postsynaptic $\mathrm{GABA}_{\mathrm{A}}$ receptor clustering in gephyrin-deficient mice. J. Neurosci. 19, 9289-9297.

Kwon, S., Chapman, E., 2011. Synaptophysin regulates the kinetics of synaptic vesicle endocytosis in central neurons. Neuron 70, 847-854.

Lamming, D., Mihaylova, M., Katajisto, P., Baar, E., Yilmaz, O., Hutchins, A., Sabatini, D., 2014. Depletion of Rictor, an essential protein component of mTORC2, decreases male lifespan. Aging Cell 13, 911-917.

Lieschke, G.J., Currie, P.D., 2007. Animal models of human disease: zebrafish swim into view. Nat. Rev. Genet. 8, 353-367.

Majdi, M., Ribeiro-da-Silva, A., Cuello, A.C., 2007. Cognitive impairment and transmitter-specific pre- and postsynaptic changes in the rat cerebral cortex during ageing. Eur. J. Neurosci. 26, 3583-3596.

Majdi, M., Ribeiro-da-Silva, A., Cuello, A.C., 2009. Systems Neuroscience: variations in excitatory and inhibitory postsynaptic protein content in rat cerebral cortex with respect to aging and cognitive status. Neuroscience 159, 896-907.

Migaud, M., Charlesworth, P., Dempster, M., Webster, L.C., Watabe, A.M., Makhinson, M., Grant, S.N., 1998. Enhanced long-term potentiation and 
impaired learning in mice with mutant postsynaptic density-95 protein. Nature 396, 433-439.

Morrison, J.H., Baxter, M.G., 2012. The ageing cortical synapse: hallmarks and implications for cognitive decline. Nat. Rev. Neurosci. 13, 240-250.

Newton, I.G., Forbes, M.E., Linville, M.C., Pang, H., Tucker, E.W., Riddle, D.R., BrunsoBechtold, J.K., 2008. Effects of aging and caloric restriction on dentate gyrus synapses and glutamate receptor subunits. Neurobiol. Aging 29, 1308-1318.

Orger, M.B., Smear, M.C., Anstis, S.M., Baier, H., 2000. Perception of Fourier and nonFourier motion by larval zebrafish. Nat. Neurosci. 3, 1128-1133.

Parichy, D.M., Elizondo, M.R., Mills, M.G., Gordon, T.N., Engeszer, R.E., 2009. Normal table of postembryonic zebrafish development: staging by externally visible anatomy of the living fish. Developmental Dyn. 238, 2975-3015.

Pavlidis, M., Digka, N., Theodoridi, A., Campo, A., Barsakis, K., Skouradakis, G., Samaras, A., Tsalafouta, A., 2013. Husbandry of zebrafish, Danio rerio, and the cortisol stress response. Zebrafish 10, 524-531.

Pinto, J., Williams, C.K., Murphy, K., Jones, D., 2015. Characterizing synaptic protein development in human visual cortex enables alignment of synaptic age with rat visual cortex. Front. Neural Circuits 9, 16.

Poe, B., Linville, C., Riddle, D., Sonntag, W., Brunso-Bechtold, J., 2001. Effects of age and insulin-like growth factor-1 on neuron and synapse numbers in area CA3 of hippocampus. Neuroscience 107, 231-238.

Prange, O., Wong, T.P., Gerrow, K., Wang, Y.T., El-Husseini, A., Nicoll, R.A., 2004. A balance between excitatory and inhibitory synapses is controlled by PSD-95 and neuroligin. Proc. Natl. Acad. Sci. U. S. A. 101, 13915-13920.

Rapp, P.R., Gallagher, M., 1996. Preserved neuron number in the hippocampus of aged rats with spatial learning deficits. Proc. Natl. Acad. Sci. U. S. A. 93, 9926-9930.

Rasmussen, T., Schliemann, T., Sorensen, J.C., Zimmer, J., West, M.J., 1996. Memory impaired aged rats: no loss of principal hippocampal and subicular neurons. Neurobiol. Aging 17, 143-147.

Ribeiro, L. Catarino, T. Santos, S., Van Leeuwen, J., Carvalho, A., Benoist, M., Esteban, J., 2014. Ghrelin triggers the synaptic incorporation of AMPA receptors in the hippocampus. Proc. Natl. Acad. Sci. U. S. A. 111, 149-158.

Rodríguez, F. López, J.C., Vargas, J.P., Gómez, Y., Broglio, C. Salas, C., 2002. Conservation of spatial memory function in the pallial forebrain of reptiles and rayfinned fishes. J. Neurosci. 22, 2894-2903.

Ruhl, T., Jonas, A., Seidel, N.I., Prinz, N., Albayram, O., Bilkei-Gorzo, A., von der Emde, G., 2015. Oxidation and cognitive impairment in the aging zebrafish. Gerontology 62, 47-57.

Saito, S., Kobayashi, S., Ohashi, Y., Igarashi, M., Komiya, Y., Ando, S., 1994. Decreased synaptic density in aged brains and its prevention by rearing under enriched environment as revealed by synaptophysin contents. J. Neurosci. Res. 39, 57-62.

Santos, E.M., Kille, P., Workman, V.L., Paull, G.C., Tyler, C.R., 2008. Sexually dimorphic gene expression in the brains of mature zebrafish. Comp. Biochem. Physiol. A. Mol. Integr. Physiol. 149, 314-324.
Sheng, M., 2001. Molecular organization of the postsynaptic specialization. Proc Natl. Acad. Sci. U. S. A. 98, 7058-7061.

Shi, L., Adams, M.M., Linville, M.C., Newton, I.G., Forbes, M.E., Long, A.B., BrunsoBechtold, J.K., 2007. Caloric restriction eliminates the aging-related decline in NMDA and AMPA receptor subunits in the rat hippocampus and induces homeostasis. Exp. Neurol. 206, 70-79.

Singleman, C., Holtzman, N., 2014. Growth and maturation in the zebrafish, Danio rerio: a staging tool for teaching and research. Zebrafish 11, 396-406.

Smith, T., Adams, M., Morrison, J., Rapp, P., Gallagher, M., 2000. Circuit-specific alterations in hippocampal synaptophysin immunoreactivity predict spatial learning impairment in aged rats. J. Neurosci. 20, 6587-6593.

Stein, V., House, D., Bredt, D., Nicoll, R., 2003. Postsynaptic density-95 mimics and occludes hippocampal long-term potentiation and enhances long-term depression. J. Neurosci. 23, 5503-5506.

Toyama, R., Jhawar, N., Aamar, E., Dawid, I., Chen, X., Epstein, J., Klein, D., 2009. Transcriptome analysis of the zebrafish pineal gland. Developmental Dyn. 238 1813-1826.

Tyagarajan, S.K., Fritschy, J., 2014. Gephyrin: a master regulator of neuronal function? Nat. Rev. Neurosci. 15, 141-156.

VanGuilder, H.D., Farley, J.A., Yan, H., Van Kirk, C.A., Mitschelen, M., Sonntag, W.E. Freeman, W.M., 2011. Hippocampal dysregulation of synaptic plasticityassociated proteins with age-related cognitive decline. Neurobiol. Dis. 43, 201-212 (Autophagy and protein degradation in neurological diseases).

VanGuilder, H.D., Yan, H., Farley, J.A., Sonntag, W.E., Freeman, W.M., 2010. Aging alters the expression of neurotransmission-regulating proteins in the hippocampal synaptoproteome. J. Neurochem. 113, 1577-1588.

Wendou, Y., De Blas, A.L., 2008. Gephyrin expression and clustering affects the size of glutamatergic synaptic contacts. J. Neurochem. 104, 830-845.

West, M.J., Slomianka, L., Gundersen, H.J., 1991. Unbiased stereological estimation of the total number of neurons in the subdivisions of the rat hippocampus using the optical fractionator. Anatomical Rec. 231, 482-497.

Wiedenmann, B., Franke, W., 1985. Identification and localization of synaptophysin, an integral membrane glycoprotein of $\mathrm{Mr} 38,000$ characteristic of presynaptic vesicles. Cell 41, 1017-1028.

Woolley, C.S., McEwen, B.S., 1993. Roles of estradiol and progesterone in regulation of hippocampal dendritic spine density during the estrous cycle in the rat. J. Comp. Neurol. 336, 293-306.

Wullimann, M.F., Rupp, B., Reichert, H., 1996. Neuroanatomy of the Zebrafish Brain: A Topological Atlas. Birkhäuser Basel, Basel.

Yang, J., Zhang, J., Chen, X., Geng, H., Ye, M., Spitzer, N., Li, X., 2013. Development of GABA circuitry of fast-spiking basket interneurons in the medial prefrontal cortex of erbb4-mutant mice. J. Neurosci. 33, 19724-19733.

Yu, L., Tucci, V., Zhdanova, I., Kishi, S., 2006. Cognitive aging in zebrafish. PLoS One 1.

Zhang, J., Konkle, A.T., Zup, S.L., McCarthy, M.M., 2008. Impact of sex and hormones on new cells in the developing rat hippocampus: a novel source of sex dimorphism? Eur. J. Neurosci. 27, 791-800. 